\title{
Energy Conservation and Pomeron Loops in High Energy Evolution
}

\author{
Gösta Gustafson \\ Lund University, Dept. of Theoretical Physics, Sölvegatan 14A, S-223 62 Lund, Sweden
}

Received on 01 November, 2006

\begin{abstract}
We present a formalism which modifies the Mueller Dipole Model such that it incorporates energymomentum conservation as well as important colour suppressed effects in the cascade evolution. The formalism is implemented in a Monte Carlo simulation program, and the results are compared to inclusive data from HERA and the Tevatron. We here find a generally very good agreement between our model and the experimental data.
\end{abstract}

Keywords: Small-x; Saturation; Multiple interactions

\section{INTRODUCTION}

To leading log or NLL accuracy the cross section for high energy $e p$ or $p p$ scattering is determined by the BFKL evolution equation. The solution corresponds to a powerlike increase for high energies, or a gluon density which grows like a power for small values of $x_{\mathrm{Bj}}$. Such a growth cannot continue indefinitely, but must be followed by a region of saturation, when the gluon density otherwise would become too high.

Saturation and rescattering is more easy to treat in transverse coordinate space than in momentum space. A dipole cascade model for small $x$ evolution has been formulated by Mueller [1-3]. This formalism leads to the leading log BFKL equation but takes also into account unitarisation effects due to multiple scatterings between partons from two colliding parton cascades. In Mueller's model dipoles in the same cascade are, however, not allowed to interact. Therefore the model does not take all saturation effects into account, and the result depends on the Lorentz frame used in the calculations.

Another problem is that Mueller's cascade is only correct to leading $\log (1 / x)$ accuracy. An important contribution to the non-leading corrections is related to energy-momentum conservation. In this talk I want to discuss an extention of Mueller's model, presented in ref. [4], which includes both effects of energy momentum conservation and saturation effects within the individual cascades before the collisions. The formalism is implemented in a MC simulation program, and I will also present some applications to DIS at HERA and to high energy $p p$ collisions.

\section{THE MUELLER DIPOLE MODEL}

In Mueller's model one starts with a $q \bar{q}$ pair, heavy enough for perturbative calculations to be applicable, and calculates the probability to emit a soft gluon from this pair. Here the quark and the antiquark are assumed to follow light-cone trajectories, and the emission of the gluon is calculated in the eikonal approximation. Adding the contributions to the emission from the quark and the antiquark, including the interference, the probability for a dipole $(\boldsymbol{x}, \boldsymbol{y})$ with endpoints in positions $\boldsymbol{x}$ and $\boldsymbol{y}$, to split into two dipoles $(\boldsymbol{x}, \boldsymbol{z})$ and $(\boldsymbol{z}, \boldsymbol{y})$ is given by

$$
\frac{d \mathcal{P}}{d Y}=\frac{\bar{\alpha}}{2 \pi} d^{2} z \frac{(\boldsymbol{x}-\boldsymbol{y})^{2}}{(\boldsymbol{x}-\boldsymbol{z})^{2}(\boldsymbol{z}-\boldsymbol{y})^{2}}
$$

Here $\boldsymbol{x}, \boldsymbol{y}$, and $\boldsymbol{z}$ are two-dimensional vectors in transverse coordinate space, $\bar{\alpha} \equiv N_{c} \alpha_{s} / \pi$, and $Y=\log (1 / x)$ denotes the rapidity, which acts as the time variable in the evolution process.

In the large $N_{c}$ limit the two new dipoles emit softer gluons independently, with the decay probability given by eq. (1). In the same limit the emission of further dipoles factorizes producing a cascade where the number of dipoles grows exponentially with $Y$ (corresponding to a power $1 / x^{\lambda}$ ).

When two dipole cascades collide, the individual cross section between two dipoles $\left(\boldsymbol{x}_{i}, \boldsymbol{y}_{i}\right)$ and $\left(\boldsymbol{x}_{j}, \boldsymbol{y}_{j}\right)$, due to single gluon exchange, is given by

$$
f_{i j}=\frac{\alpha_{s}^{2}}{8} \ln ^{2}\left(\frac{\left(\boldsymbol{x}_{i}-\boldsymbol{x}_{j}\right)^{2}\left(\boldsymbol{y}_{i}-\boldsymbol{y}_{j}\right)^{2}}{\left(\boldsymbol{x}_{i}-\boldsymbol{y}_{j}\right)^{2}\left(\boldsymbol{y}_{i}-\boldsymbol{x}_{j}\right)^{2}}\right)
$$

The sum $\sum f_{i j}$ then corresponds to single pomeron exchange. At high energies $\sum f_{i j}$ can be large, and in an eikonal approximation the contributions from multiple pomeron exchange exponentiates and the cross section saturates.

\section{ENERGY MOMENTUM CONSERVATION}

We note that the expression in eq. (1) has non-integrable singularities at $z=x$ and $z=y$. In numerical calculations it is therefore necessary to introduce a cutoff, $\rho$, such that $(\boldsymbol{x}-\boldsymbol{z})^{2},(\boldsymbol{z}-\boldsymbol{y})^{2} \geq \rho^{2}$. The scattering probability for small dipoles is, however, suppressed in such a way that the total cross section has a finite limit when $\rho \rightarrow 0$.

A small dipole means that we have two well localized gluons in the transverse plane, and these gluons must then have a correspondingly large transverse momentum. If these small dipoles are interpreted as corresponding to real emissions with $p_{\perp} \sim 1 / r$, it would imply a severe violation of energymomentum conservation. Therefore such dipoles have to be interpreted as virtual fluctuations, and will not be present in exclusive final states.

It is earlier realized that energy conservation has an important effect on the evolution of parton cascades [5]. In ref. [6] we presented a formalism to take into account energymomentum conservation in Mueller's model, using the Linked Dipole Chain (LDC) model [7] as a guidance. 


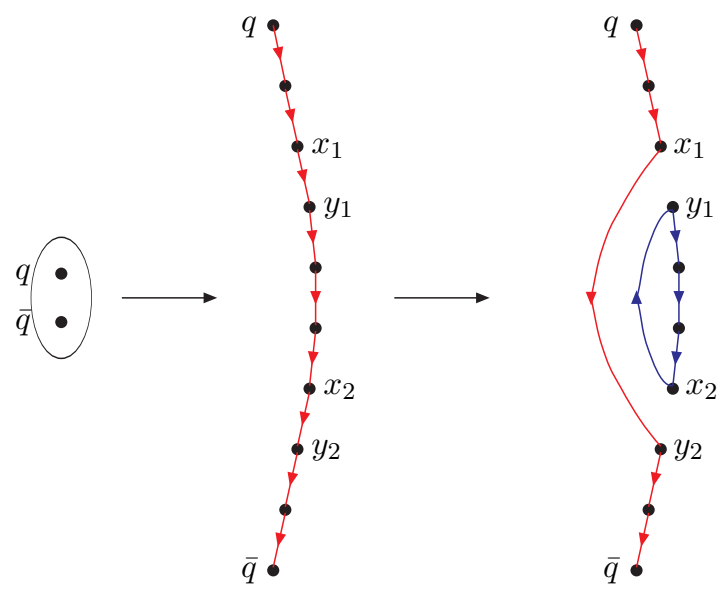

FIG. 1: Schematic picture of a colour recoupling, or dipole swing. The two dipoles $\left(\boldsymbol{x}_{1}, \boldsymbol{y}_{1}\right)$ and $\left(\boldsymbol{x}_{2}, \boldsymbol{y}_{2}\right)$ are transformed into two new dipoles $\left(\boldsymbol{x}_{1}, \boldsymbol{y}_{2}\right)$ and $\left(\boldsymbol{x}_{2}, \boldsymbol{y}_{1}\right)$ after a recoupling of the colour flow. The initial chain of dipoles is replaced by a new chain stretching between the original $q \bar{q}$ pair, red colour, and a loop of dipoles, blue colour.

We used this formalism in a MC program and found that the number of dipoles grows much more slowly, and the onset of saturation is correspondingly delayed. In fact it is found that in DIS the unitarity effects become quite small within the HERA energy regime.

An important consequence of energy-momentum conservation is that it implies a dynamical cutoff, $\rho(\Delta y)$, which is large for small steps in rapidity, but gets smaller for larger steps $\Delta y$. Besides its physical effects reducing the growing gluon density, energy-momentum conservation also simplifies the MC treatment, since large numerical complications in a $\mathrm{MC}$ without energy conservation, as discussed in [8], are not present.

\section{FINITE $N_{c}$ EFFECTS IN DIPOLE LANGUAGE}

As mentioned above, in Mueller's dipole model saturation effects are included from multiple interactions in a collisions between two cascades, but not in the evolution of each cascade separately. The multiple dipole-dipole interactions due to gluon exchange can give rise to closed loops of colour dipoles, and in a Lorentz invariant formalism such loops must be possible also within the individual parton cascades. The dipoledipole scattering in eq. (2) is proportional to $\alpha_{s}^{2}$, and therefore formally colour suppressed compared to the dipole splitting process in eq. (1), which is proportional to $\bar{\alpha}=N_{c} \alpha_{s} / \pi$. A mechanism giving dipole loops within the evolution cascade must therefore also be colour suppressed, and thus could not be included in Mueller's cascade, which is only exact to leading order in $N_{c}$.

The dipole loops are related to triple pomeron couplings and to the pomeron merging process. Several attempts have been presented to describe pomeron merging within the dipole formalism, see e.g. refs. $[9,10]$

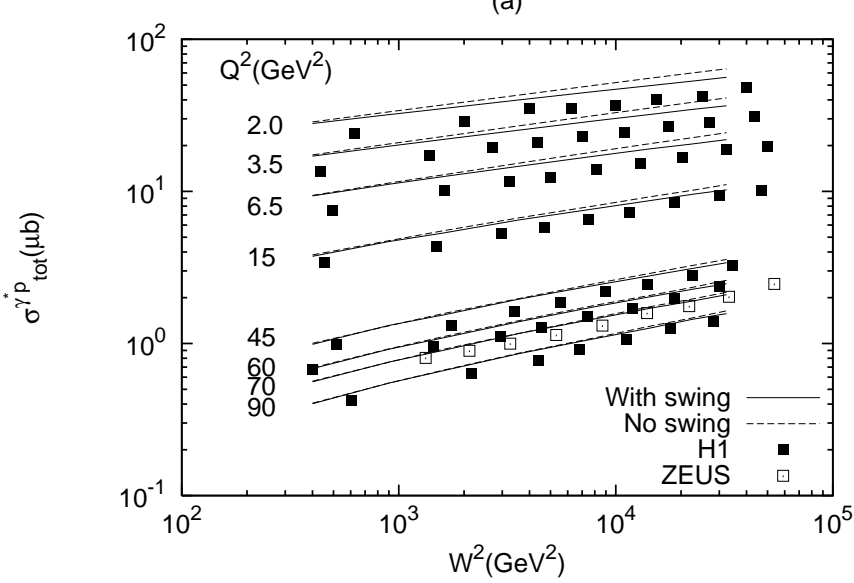

FIG. 2: The $\gamma^{*} p$ total cross section shown for different $Q^{2}$. The solid lines include the dipole swing while the dashed lines are without the dipole swing. Data points are taken from $[11,12]$.

(b)

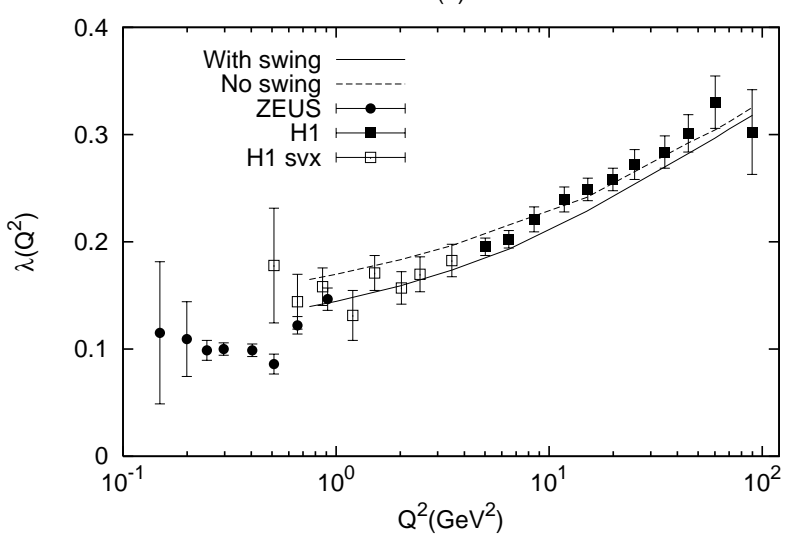

FIG. 3: The effective slope measured at different $Q^{2}$. The solid line is our result including the dipole swing while the dashed line is without. Filled circles are data from ZEUS[12] while filled[11] and open[13] squares are data from $\mathrm{H} 1$.

With a finite number of colours also colour quadrupoles and higher multipoles can be produced in the evolution. This cannot be described exactly in a factorized form, but it may be possible to find an improved approximation to the colour field in terms of two dipoles formed by nearby charge-anticharge pairs. This will give rise to a recoupling of the dipoles in the dipole chain corresponding to a "dipole swing" as illustrated in Fig. 1. Such a swing could also be caused by gluon exchange inside the cascade, and it will result in closed loops in the dipole chains. In ref. [4] we studied a formalism where loops can be formed by such a swing, with a probability which is suppressed by a factor $\sim 1 / N_{c}^{2}$. The strength of this recoupling should be such that the result is Lorentz frame independent. 


\section{RESULTS}

Our formalism has been applied to $\gamma^{*} p$ and $p p$ scattering using a MC simulation program. The coupling of a virtual photon to a $q \bar{q}$ dipole is well known. The proton structure has to be modelled, and is in our calculations represented by three dipoles in a triangular configuration.

The results for DIS are presented in Figs. 2 and 3. Fig. 2 shows the total cross section and Fig. 3 shows the result for the logarithmic slope, $\lambda_{\text {eff }}=d(\log \sigma) / d(\log 1 / x)$, for different values of $Q^{2}$. We see that there is a generally good agreement between the model and the experimental data for all points in the interval $1 \mathrm{GeV}^{2} \lesssim Q^{2} \lesssim 100 \mathrm{GeV}^{2}$. We also see that the effect of saturation is small, and that including the swing gives only a minor correction.

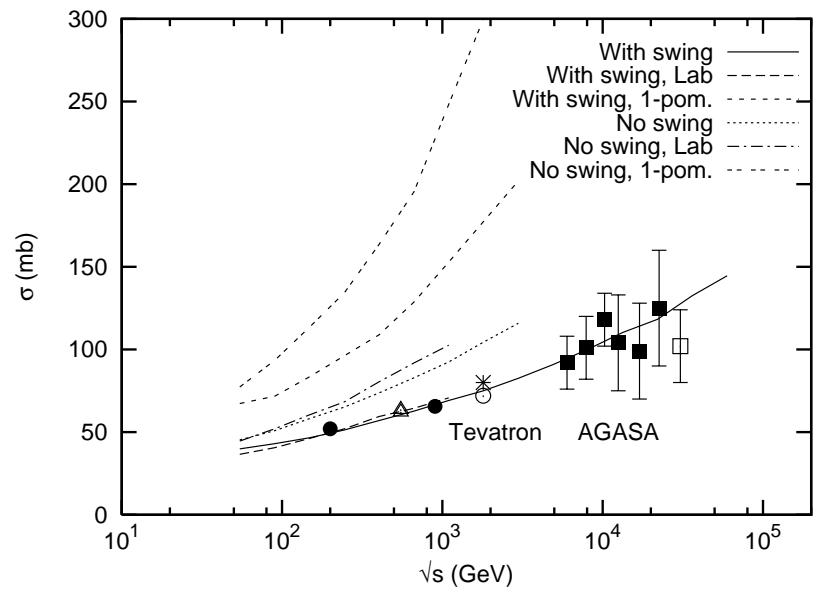

FIG. 4: The total cross section for $p p$ scattering as a function of the cms energy $\sqrt{s}$. Here results are shown for evolution with and without the dipole swing mechanism. The results for the one pomeron cross sections are also shown. Also shown are the results obtained in the "lab" frame where one of the protons is almost at rest.
Results for the $p p$ total cross section are shown in Fig. 4. Here the saturation effects from multiple scattering and the dipole swing are much larger. The figure also shows results obtained in a frame where one of the protons is at rest. It was stressed above that the saturation effects in the cascade have to imply that the result is frame independent. In Fig. 4 we note especially that including the swing indeed makes the result practically independent of the Lorentz frame.

\section{CONCLUSIONS}

A formalism is presented to include energy conservation and saturation effects corresponding to pomeron merging in Mueller's dipole cascade model. Results from MC simulations agree well with experimental data for DIS and $p p$ total cross sections. We conclude that although we do not yet have an explicitely frame independent formalism, it appears as if our implementation of the dipole swing does include the most essential features of the pomeron merging process. Our formalism is, however, still only applicable for total cross sections. The energy conservation constraint does reduce the number of virtual dipoles, but they are not totally eliminated. In future work we want to develop the formalism further in order to also be able to describe the properties of exclusive final states.
[1] A. H. Mueller, Nucl. Phys. B 415, 373 (1994).

[2] A. H. Mueller and B. Patel, Nucl. Phys. B 425, 471 (1994), $\{$ hep-ph/9403256\}

[3] A. H. Mueller, Nucl. Phys. B 437, 107 (1995), \{hep-ph/ 9408245\}.

[4] E. Avsar, G. Gustafson, and L. Lönnblad, JHEP 0701, 012 (2007).

[5] J. R. Andersen, Phys. Lett. B 639, 290 (2006), \{hep-ph/ 0602182\}.

[6] E. Avsar, G. Gustafson, and L. Lönnblad, JHEP 07, 062 (2005), \{hep-ph/0503181\}

[7] B. Andersson, G. Gustafson, and J. Samuelsson, Nucl. Phys. B 467, 443 (1996).

[8] G. P. Salam, Nucl. Phys. B 461, 512 (1996), \{hep-ph/
9509353\} .

[9] E. Iancu, G. Soyez, and D. N. Triantafyllopoulos, Nucl. Phys. A 768, 194 (2006), \{hep-ph/0510094\}

[10] E. Levin and M. Lublinsky, Michael, Nucl. Phys. A 763, 172 (2005), \{hep-ph/0501173\}

[11] H1 Collaboration, C. Adloff et al., Eur. Phys. J. C 21, 33 (2001), \{hep-ex/0012053\}

[12] ZEUS Collaboration, J. Breitweg et al., Phys. Lett. B 487, 53 (2000), \{hep-ex/0005018\}.

[13] A. Petrukhin, "New Measurement of the Structure Function $F_{2}\left(x, Q^{2}\right)$ at low $Q^{2}$ with Initial State Radiation Data." Proceedings of DIS04, Štrbské Pleso, Slovakia, 2004. 